\title{
A metodologia das maratonas de programação em um projeto de extensão: um relato de experiência
}

\author{
Ana Elisa Tozetto Piekarski ${ }^{1}$, Mauro Miazaki ${ }^{1}$, Tony Alexander Hild ${ }^{1}$, \\ Mauro Henrique Mulati ${ }^{1}$, Daniel Kikuti \\ ${ }^{1}$ Departamento de Ciência da Computação - Universidade Estadual do Centro-Oeste \\ (UNICENTRO) \\ Rua Simeão Camargo Varela de Sá, 03 - Vila Carli - 85.040-080 - Guarapuava, PR - \\ Brazil \\ ${ }^{2}$ Departamento de Informática - Universidade Estadual de Maringá (UEM) \\ Maringá, PR - Brazil \\ \{aetpiekarski,maurom, tony.hild,mhmulati\}@gmail.com, dkikuti@din.uem.br
}

\begin{abstract}
This paper describes the experience of an extension project for the training in computer programming, using the methodology of programming contest and aiming at the selection of teams to participate in the First Phase of the SBC Programming Contest. In addition to describing the carried out activities and present some project results, this paper aims to share the adopted methodology, in order to discuss possibilities and strategies for future activities.
\end{abstract}

\begin{abstract}
Resumo. Este artigo descreve a experiência de um projeto de extensão destinado ao treinamento em programação de computadores, utilizando a metodologia das maratonas de programação e visando a seleção de times para participação na Primeira Fase da Maratona de Programação da SBC. Além de descrever as atividades realizadas e apresentar alguns resultados do projeto, o artigo visa compartilhar a metodologia adotada, a fim de discutir possibilidades e estratégias para atividades futuras.
\end{abstract}

\section{Introdução}

O Departamento de Ciência da Computação da Unicentro sempre estimulou seus alunos a participar da Primeira Fase da Maratona de Programação da Sociedade Brasileira de Computação (SBC) ${ }^{1}$, oferecendo a inscrição e o transporte dos times selecionados. Para a seleção dos times, um grupo de professores se reunia para preparar e aplicar uma prova aos interessados, elaborada com problemas de maratonas anteriores, e alguns participavam como coach (técnico) dos times.

A partir de 2011, foi proposto um projeto de extensão com o objetivo geral de oferecer oportunidades extracurriculares de treinamento em Programação de Computadores aos alunos do Bacharelado em Ciência da Computação e demais acadêmicos interessados, tendo como maior enfoque a preparação e seleção de times para participar da Primeira Fase da Maratona de Programação.

\footnotetext{
${ }^{1}$ http://maratona.ime.usp.br
} 
Considerando a pertinência das maratonas como metodologia de treinamento em programação de computadores, bem como todo o arcabouço conceitual e as ferramentas associadas, e que outras iniciativas são realizadas com objetivos similiares em várias instituições, este artigo descreve a metodologia adotada no projeto, os resultados obtidos e as dificuldades enfrentadas em quatro anos de execução, a fim de compartilhar a experiência do projeto e discutir desdobramentos futuros.

\section{As maratonas de programação: aspectos metodológicos}

As maratonas de programação constituem uma metodologia já difundida e bastante utilizada em todo o mundo. Em 1970, teve início uma competição de programação na Universidade do Texas, que alguns anos mais tarde deu lugar à ICPC (International Collegiate Programming Contest), organizada pela ACM (Association for Computer Machinery) e promovida pela IBM, que passou a acontecer na Universidade de Baylor. A competição ocorre em etapas desde 1977, no início envolvia apenas times dos EUA e Canadá $^{2}$. De setembro a dezembro de 2014, as competições regionais envolveram mais de 38 mil alunos, de mais de 2.500 instituições, em 101 países, selecionando 128 times para a final mundial. Esses números representam o volume recorde de envolvidos até então, ano a ano a competição mantém seu crescimento ${ }^{3}$.

A ICPC "estimula criatividade, trabalho em equipe e inovação no desenvolvimento de novos programas de software, possibilitando aos estudantes testar suas habilidades de trabalhar sobre pressão. Resumindo, é a mais antiga, maior e mais prestigiada competição de programação no mundo."4

O Brasil participa da ICPC desde 1996. A partir de 2000, a Sociedade Brasileira de Computação (SBC) começou a promover a Maratona de Programação como seleção dos times para a final mundial [De Campos e Ferreira 2004]. A Maratona de Programação também é realizada em etapas (primeira fase e final nacional). Para participar, as equipes devem ter três competidores, que, utilizando um único computador e consulta a materiais impressos, se revezam entre desenvolver a lógica de resolução dos problemas, codificar o programa e testar.

A metodologia das maratonas de programação reúne características de três abordagens de aprendizado distintas, mas que podem ser aplicadas de forma complementar: cooperativa, competitiva e baseada em problemas.

O aprendizado cooperativo é constituído por técnicas de ensino em que os estudantes trabalham em atividades de aprendizado em pequenos grupos e recebem gratificações e reconhecimento baseado no desempenho do grupo [Slavin 1980]. A ideia é ampliar a troca de informações e conhecimentos entre os estudantes para motivar seu próprio aprendizado e estimular uns aos outros [Slavin 1980], [Attle e Baker 2007], [Burguillo 2010].

O aprendizado baseado em competição é uma metodologia onde o aprendizado é ativado por meio da competição, mas o resultado do aprendizado é independente do

\footnotetext{
${ }^{2}$ http://icpc.baylor.edu/

${ }^{3}$ icpc.baylor.edu/worldfinals/pdf/Factsheet.pdf

${ }^{4}$ http://icpc.baylor.edu/
} 
resultado da competição (classificação obtida pelo estudante) [Burguillo 2010]. A relação entre desempenho e resultado são dependentes do padrão competitivo adotado [Slavin 1980].

O aprendizado baseado em problemas resulta do processo de trabalhar através da compreensão ou resolução de um problema; o ponto de partida para o aprendizado é o problema [Attle e Baker 2007].

O método de resolução de problemas proposto por Polya (1945) envolve quatro princípios básicos: a compreensão do problema, percebendo claramente o que é necessário; o estabelecimento de um plano, observando a relação dos dados e modelando a solução; a execução do plano; e a retrospectiva da resolução, revendo e discutindo, para que ocorra a consolidação do conhecimento.

Nas maratonas, os estudantes que compõem um time devem colaborar uns com os outros, a fim de que o time obtenha um bom resultado. Nessas interações, os estudantes discutem os problemas (compreensão do enunciado), as possibilidades de resolução (arcabouço conceitual necessário), aspectos técnicos (relacionados ao ambiente computacional e à linguagem de programação utilizada), ampliando seus conhecimentos e as habilidades de trabalho em equipe.

Sob o aspecto da colaboração entre os estudantes especificamente nas maratonas, Trotman e Handley (2008) discutem estratégias para maximizar o desempenho do time. Analisando as várias estratégias de trabalho dos times (trabalho em equipe, trabalho isolado dos membros, trabalho em pares) e a ordem em que os problemas devem ser resolvidos (ordem numérica, os mais fáceis primeiro, os mais difíceis primeiro, alternar entre fáceis e difíceis, os de nível médio de dificuldade primeiro, entre outras), os autores propõem que os times devem considerar as possibilidades de sucesso quando iniciam uma competição; pois o conjunto dos problemas e as estratégias dos outros times afetam suas possibilidades. De modo geral, usando um conjunto variado de estratégias o time maximiza suas chances de sucesso.

Sobre o aspecto competitivo, a disputa entre os times faz com que os estudantes se esforcem em obter respostas positivas para as soluções que o time submete, buscando aplicar o arcabouço conceitual de que dispõem e mesmo buscar novos conhecimentos (por meio de pesquisas ao material de consulta). Ratificando o aspecto metodológico do aprendizado baseado em competição, não é possível mensurar o aprendizado de cada time (ou isoladamente de seus membros) a partir dos resultados da competição.

Para Attle e Baker (2007), a oportunidade instrutiva de oferecer atividades de aprendizado em que os estudantes cooperam em times que competem uns com os outros maximiza o aprendizado dos estudantes e seu desenvolvimento profissional, pois trata-se de um ambiente similar ao mundo corporativo. "Estruturar um projeto competitivo baseado em problemas onde times de estudantes competem uns com os outros, é uma forma do instrutor poder assegurar oportunidades de aprendizado cooperativas e competitivas aos estudantes" [Attle e Baker 2007 p.78].

Outra característica da metodologia das maratonas que merece atenção é o enunciado dos problemas. De acordo com Zanini e Raabe (2012), na bibliografia adotada em disciplinas iniciais de programação em 51 cursos de graduação do Brasil, os enunciados dos exercícios não estão inseridos em um contexto $(64,72 \%$ dos 
enunciados), e, dos enunciados que possuem contexto, $55 \%$ estão em contexto matemático, sendo essas as dificuldades dos alunos, e não propriamente a lógica algoritmica.

De forma oposta, os problemas das maratonas são muito bem contextualizados, em consonância com a metodologia de resolução de problemas. Os limites das instâncias são definidos nos enunciados, permitindo que os times imprimam esforços na solução do problema em si. Por outro lado, se os competidores não estão acostumados a esse tipo de enunciado, o treinamento é necessário para que sejam capazes de extrair as informações pertinentes à resolução daquelas que simplesmente compõem o cenário do problema.

Programação de computadores é um dos conteúdos mais complexos da área de Computação, constituindo uma das bases mais importantes para a futura atuação profissional dos estudantes. O grau de dificuldade desses conteúdos se reflete na quantidade de desistências ao longo da disciplina e no alto índice de reprovação.

A fim de motivar os alunos para o aprendizado, deve-se buscar novas atividades de caráter pedagógico. Nesse sentido, as maratonas são promissoras, pois permitem aos alunos aplicar conceitos de desenvolvimento de software, desenvolver habilidades de resolução de problemas e adquirir experiência em programação de computadores, incluindo linguagens, técnicas e ferramentas (ambientes). Iniciativas nesse contexto são realizadas em diversas IES.

Na Universidade Federal do Rio de Janeiro (UFRJ) é ofertada a disciplina Tópicos Especiais em Programação, que visa: estimular o raciocínio e a criatividade para a resolução de problemas (quase) reais, aproximando teoria e prática; despertar interesse em competições de resolução de problemas e programação; e treinar para a Maratona de Programação [Mota e Santos 2009].

Motivados a buscar uma alternativa para estimular o aprendizado de programação de computadores, Oliveira, Paula e Dias (2012) relatam sua experiência no IFSULDEMINAS, realizando competições de programação. A experiência, ainda em fase inicial, se propõem a coletar os dados dos alunos dos vários cursos da área que a instituição oferece, a fim de futuramente analisar o impacto da atividade na motivação dos alunos para continuidade no curso (e na área).

\section{As atividades do projeto}

Para atingir os objetivos propostos, o projeto contempla uma série de atividades: encontros ordinários para a discussão e resolução de problemas, competições simuladas, provas de seleção dos times para competir na Primeira Fase da Maratona de Programação representando a instituição, e preparação dos times selecionados.

Os encontros são desenvolvidos visando oferecer atividades lúdicas que favoreçam a aprendizagem e/ou o aperfeiçoamento das habilidades em Programação de Computadores. A ideia seria balancear um conteúdo conceitual (revisão/explicação de estrutura de dados, grafos, técnicas de programação, ...) com uma série de problemas de competições passadas (exercícios), disponibilizados no ambiente de avaliação automática para que os times treinem e testem suas soluções. Também é utilizado um ambiente virtual de aprendizagem (Moodle), onde são compartilhados materiais com os 
conceitos discutidos nos encontros e de apoio. As principais fontes bibliográficas são Skiena e Revilla (2003), conforme apontado em Trotman e Handley (2008) como uma das fontes mais largamente utilizadas nesses treinamentos, e Arefin (2006).

As competições seguem o mesmo modelo das maratonas: os times resolvem os problemas propostos e os submetem à avaliação por meio do ambiente de julgamento automático denominado BOCA. O BOCA, que é utilizado nas competições oficiais da SBC, permite que os times previamente cadastrados submetam as soluções para os problemas propostos, tenham suas soluções avaliadas de forma automatizada e acompanhem a classificação dos times participantes em tempo real [De Campos e Ferreira 2004]. Quando submete um solução, o time obtém uma resposta do sistema. Se a solução estiver correta, o time é notificado e recebe um balão (físico) com a cor do problema (cada problema é associado a uma cor), o que contribui para estimular os times na competição, além de tornar o espaço físico mais descontraído. Se a solução estiver incorreta, o time é notificado em um dos seguintes aspectos: erro de apresentação dos dados (Presentation Error), resposta errada (Wrong Answer), erro de compilação (Compilation Error), tempo de execução excedido (Time-limit Exceeded), erro em tempo de execução (Runtime Error). Embora as respostas do ambiente sejam bastante sintéticas, os times podem identificar o erro, corrigir e submeter nova solução.

Outra atividade do projeto é a "Gincana de Programação", destinada aos alunos da disciplina introdutória de Programação de Computadores, no primeiro semestre do curso. Nos moldes das competições, a prova é elaborada de acordo com o conteúdo trabalhado na disciplina. Além de propiciar uma atividade diferente aos alunos da disciplina, com todos os aspectos metodológicos de aprendizado para testar seus conhecimentos (tanto em relação à linguagem quanto à lógica de programação), os estimula para a participação futura em outras atividades do projeto.

No primeiro ano que o projeto foi ofertado, a seletiva dos times para a Primeira Fase da Maratona seguiu a mesma programação, tendo uma fase de preparação antes da prova. Na fase de preparação, a equipe executora do projeto explanou sobre as competições de programação, o formato dos problemas, as funções de entrada e saída de dados, o ambiente utilizado nas competições (BOCA), as ferramentas de apoio para treinamento e exercícios de aquecimento. Nas ofertas seguintes, inclusive por restrições de disponibilidade de tempo, apenas a prova foi realizada.

A prova, tendo em média cinco problemas, é elaborada em dois níveis: Iniciante (para os alunos da primeira série do curso) e Intermediário (para os demais alunos, inclusive participantes do projeto). São três horas de prova, a fim de selecionar os times com melhor desempenho.

Aos times selecionados, são oferecidos encontros para um treinamento mais focado, que inclui ajustes nas inscrições dos times, orientações para minimizar as 
dificuldades dos anos anteriores ${ }^{5}$ e apoio para preparação do material de consulta que poderão utilizar na competição.

A partir de 2014, o projeto teve um ganho importante, pois foi estabelecida uma parceria com o Departamento de Informática da UEM, permitindo a realização de atividades conjuntas. Além da escolha de problemas a serem trabalhados nos encontros e da elaboração das provas, a seletiva dos times para a Primeira Fase da Maratona de Programação em 2014 foi realizada simultaneamente para os times das duas IES. Utilizando o mesmo ambiente de apoio (BOCA) com acesso remoto, os times das duas universidades acompanharam o desempenho uns dos outros e puderam experimentar uma competição, como preparação para a prova a que estavam sendo selecionados.

\section{Discussões}

A forma como se desenvolveram os encontros indica que este ainda é um desafio a ser enfrentado na continuação do projeto. Inicialmente sempre há uma boa demanda de participantes, mas a medida em que o projeto vai se desenvolvendo, percebe-se uma alta taxa de abandono. Este não é um problema isolado. Em outras instituições, em projetos similares, enfrentam-se os mesmos problemas.

Ferrasa e Souza (2012) comentam o desinteresse dos alunos que participam de um projeto similar, listando os seguintes motivos: as responsabilidades das disciplinas do curso e de outras atividades desenvolvidas pelos alunos acabam diminuindo o tempo que eles podem se dedicar ao treinamento; a crescente dificuldade dos problemas, que ficavam sem solução; e a inexperiência do professor que estava conduzindo o treinamento.

A fim de avaliar a continuidade dos participantes no projeto, foram tabulados os dados das atividades (encontros, seletivas, gincanas) realizadas durante o período de 2011 a 2014, totalizando onze atividades. Ao todo, foram 81 alunos participantes das diferentes atividades. Utilizando o critério de participação em mais de uma atividade ao longo do período analisado, foram 34 alunos envolvidos (42\%), dos quais:

- 7 participaram em mais de uma atividade no mesmo ano;

- 8 participaram em mais de uma atividade em dois anos distintos;

- 2 participaram em mais de uma atividade em três anos distintos;

- 13 participaram nos quatro anos do projeto (desde o primeiro ano do curso);

- 4 participaram desde que o projeto começou até o fim do curso (2 alunos em dois anos e 2 em três anos do projeto).

Assim, é significativo que, de todos os participantes, $21 \%$ dos que tiveram algum contato com o projeto continuaram participando ativamente das atividades oferecidas.

\footnotetext{
${ }^{5}$ Em 2011, os participantes da Primeira Fase apontaram as seguintes dificuldades: cansaço da viagem (distância deslocada e horário de saída inconveniente), desconhecimento do uso dos recursos do ambiente (como impressão de código submetido) e falta de informação sobre as regras da competição (funcionamento de penalidades por submissões erradas). Esses aspectos foram trabalhados pela equipe de coordenação e, nos anos seguintes, as dificuldades estavam relacionadas a complexidade dos problemas (a solução implementada não atendia a todos os casos de teste ou esbarrava no tempo limite de execução).
} 
O que tem acontecido é que os times que participam tendem a continuar nos anos seguintes. Nesse sentido, é salutar estimular alunos desde o primeiro ano: dos ingressantes de 2011 que participaram de atividades do projeto, 75\% voltaram a participar nos anos seguintes; o time de calouros que participou da comitiva na Primeira Fase em 2013 participou de forma ativa do projeto em 2014; em 2014, tivemos alguns alunos calouros que participaram dos encontros iniciais, mas apenas um time foi formado para disputar a seletiva e não obteve desempenho para se classificar.

Uma das dificuldades encontradas no decorrer do projeto foi a de propiciar a interação de alunos de séries diferentes em atividades de resolução de problemas extra-classe. Na maioria, os times são constituídos por alunos da mesma série. Alguns problemas exigem o domínio de estrutura de dados e técnicas de programação que alunos dos primeiros anos não possuem. Sem esta bagagem, os assuntos mais complexos tornam-se desinteressantes e nem chegam a constituir um desafio. A possibilidade de realizar encontros preparatórios com conteúdos diferenciados de acordo com a maturidade dos alunos pode ser explorada quando da disponibilidade da equipe de coordenação e de intervalos no horário dos alunos.

A grade horária do curso em que o projeto está inserido é cheia nos períodos diurnos, sendo difícil encontrar uma janela em que todas as turmas (preferencialmente a partir do segundo ano) tenham disponibilidade. No período noturno, muitos alunos assumem outros compromissos, como estágios e aulas não curriculares. Infelizmente, é uma questão administrativa que foge do alcance da coordenação do projeto.

Outra dificuldade que tem sido contornada é a infraestrutura para o ambiente Boca. O projeto tem disponível uma máquina que atende razoavelmente a necessidade computacional do sistema. No entanto, o acesso externo é frequentemente comprometido, bem como a manutenção do fornecimento elétrico. Algumas alternativas quanto ao ambiente estão sendo discutidas e testadas.

Assim como destacado por Burguillo (2010) sobre a abordagem competitiva, podemos observar os seguintes aspectos como estímulo ao aprendizado:

- Embora a abordagem seja orientada à participação em competições, o aprendizado independe da classificação obtida na competição;

- A competição é executada entre diferentes grupos, mas internamente os estudantes do grupo devem colaborar para melhorar suas possibilidades;

- A abordagem pode ser facilmente integrada com outras metodologias de aprendizado, que complementem suas vantagens particulares.

\section{Considerações finais}

De modo geral, o conjunto das atividades realizadas no âmbito do projeto permitiu:

- Despertar nos alunos o interesse pelos problemas das competições de programação;

- Desenvolver/incrementar o conhecimento dos participantes em linguagens de programação e suas bibliotecas;

- Estimular a resolução de problemas computacionais;

- Estimular o trabalho em equipe; 
CBIE-LACLO 2015

Anais dos Workshops do IV Congresso Brasileiro de Informática na Educação (CBIE 2015)

- Incentivar a participação nas Maratonas de Programação.

Como resultados quantitativos, no biênio 2011-2012, destaca-se o envolvimento de 50 discentes nas diferentes atividades do projeto e a participação de dois times na Primeira Fase da Maratona da SBC em 2011 e três times em 2012, sendo que um deles foi o primeiro colocado na sub-sede em que competiu. No biênio 2013-2014, 51 discentes participaram das diferentes atividades do projeto. Dois times participaram na Primeira Fase em 2013 e três times em 2014. Em todo o período, 20\% de taxa de permanência no projeto por parte dos participantes é um valor significativo. Na continuidade do projeto, o acompanhamento das participações fornecerá subsídios para discussões e melhorias nas atividades oferecidas.

O projeto iniciou tendo como base a experiência do que vinha sendo realizado e em iniciativas semelhantes em outras IES, voltado às atividades práticas. Com o tempo, vimos sentindo necessidade de ampliar o arcabouço teórico-metodológico, a fim de propiciar melhores experiências de aprendizado aos participantes, bem como ampliar - e poder mensurar - os resultados do projeto. No entanto, o aspecto que julgamos mais crítico, ainda sujeito a investigação e discussão, é a avaliação dos ganhos por parte dos participantes. Se, simultaneamente às atividades do projeto, os alunos cursam disciplinas e se envolvem em outros projetos de cunho científico, qual a origem dos conteúdos aprendidos? O mais trivial seria avaliar o desempenho dos times no decorrer do projeto, mas o embasamento metodológico nos diz que não é possível mensurar o aprendizado dos times ou de seus membros a partir dos resultados da competição.

Assim, ao relatar essa experiência, buscamos compartilhar, discutir e ampliar as possibilidades metodológicas, planejamento das ações futuras, formas de acompanhamento do projeto e de mensuração do aprendizado dos envolvidos.

\section{Referências}

Arefin, A. S. (2006). Art of Programming Contest, Gyankosh Prokashoni, $2^{\text {nd }}$ edition.

Attle, S. and Baker, B. (2007). Cooperative learning in a competitive environment: classroom applications. International Journal of Teaching and Learning in Higher Education, 19(1):77-83.

Burguillo, J. C. (2010). Using game theory and Competition-based Learning to stimulate student motivation and performance. Computers \& Education, 55(2):566-575.

De Campos, C. P. and Ferreira, C. E. (2004). BOCA: um sistema de apoio a competições de programação. In Anais do XXIV Congresso da Sociedade Brasileira de Computação, XII WEI - Workshop de Educação em Computação, Salvador.

Ferrasa, M. and Souza, M. A. (2012). Competições de raciocínio lógico e programação de computadores: um relato de experiência. In Anais do $10^{\circ}$ CONEX - Conversando Sobre Extensão, Ponta Grossa. 
CBIE-LACLO 2015

Anais dos Workshops do IV Congresso Brasileiro de Informática na Educação (CBIE 2015)

Mota, T. C. and Santos, V. F. (2009). Tópicos especiais em programação: apresentação da disciplina. Disponível em: <http://maratona.dcc.ufrj.br/tep/material/aula1.1.pdf $>$. Acesso em: 12 de jul. de 2015.

Oliveira, A., Paula, L., and Dias, J. C. (2012). Experiências no estímulo à prática de programação através do desenvolvimento de atividades extracurriculares relacionadas com as competições de conhecimentos. In Anais do XXXII Congresso da Sociedade Brasileira de Computação, XX WEI - Workshop sobre Educação em Computação, Curitiba.

Polya, G. (1945). How to solve it. Princeton University, Princeton.

Skiena, S. S. and Revilla, M. (2003). Programming challenges. Springer-Verlag, New York.

Slavin, R. E. (1980). Cooperative learning. Review of Educational Research, $50(2): 315-342$.

Trotman, A. and Handley, C. (2008). Programming contest strategy. Computers \& Education, 50(3):821-837.

Zanini, A. S. and Raabe, A. (2012). Análise dos enunciados utilizados nos problemas de programação introdutória em cursos de Ciência da Computação no Brasil. In Anais do XXXII Congresso da Sociedade Brasileira de Computação, XX WEI - Workshop sobre Educação em Computação, Curitiba. 